\title{
Editor's Message to Special issue of Computer Security Technologies to be Indispensable to the Society
}

\author{
Masahiro Mambo ${ }^{1}$
}

Information and Communications Technology (ICT) such as the Internet has become indispensable to our daily life. Recently, many incidents have occurred which indicate that ICT cannot be discussed without security technologies as follows.

In the personal data leakage from Japan Pension Service occurred in 2015, it has been reported that targeted email attacks have been conducted, where emails with a service-related subject line were sent to employee and their computers were infected by a malicious program. The infected computers were remotely controlled and data were stolen from penetrated networks. In addition, personal data were stored not in a physically different network as determined by its security policy but in an easily accessible network. The incident gives us a chance to pay attention not only to technical problems of computer system but also to management problem and even praxeology or social science related problems.

The massive personal data leakage from a major correspondence education provider in Japan has been conducted by a temporary staff of subcontractor during his work inside the provider. The incident makes us re-recognize the need of security countermeasure enough incorporating human factors in system management.

The widespread use of various ICT in our life means that computer security technologies are also used in various situations and become indispensable to the society. This special issue aims to identify technical problems, management problems and even problems related to praxeology or social science and to understand existing/possible solutions to these problems. We apply such knowledge to future research, development and management.

Out of 62 submissions, 11 and 19 regular papers written in English and Japanese, respectively, were accepted (acceptance rate: $48 \%$ ). Details of number of accepted papers are as follows. Computer network: 13, System security: 10 and Security and society: 6 and so on. Unlike previous tendency, there are less papers on fundamental primitives and more papers on security of computer network and system.

The special issue includes an invited paper, A Model for Adversarial Wiretap Channels and its Applications by Reihaneh Safavi-Naini and Pengwei Wang, which was the keynote of IWSEC 2014. We would like to show our sincere appreciation to their contribution.

Finally, we would like to sincerely thank all reviewers, editors and secretaries of IPSJ for their dedication to select excellent papers and publish the special section. Especially, we appreciate the work of editorial secretaries, Takao Okubo (Inst. of Information Security) and Isao Echizen (National Inst. of Informatics). Without their work, we could not publish the special issue.

\section{The Editorial Committee}

- Editor in-Chief:

Masahiro Mambo (Kanazawa Univ.)

- Editorial Board:

Takao Okubo (Inst. of Information Security), Isao Echizen (National Inst. of Informatics)

\section{- Editorial Committee:}

Koji Chida (NTT Corporation), Hiroshi Doi (Inst. of Information Security), Sadayuki Hongo (Hokkaido Inst. of Tech.), Keiichi Iwamura (Tokyo Univ. of Science), Takehisa Kato (IPA), Hiroaki Kikuchi (Meiji Univ.), Takashi Matsunaka (KDDI R\&D Laboratories Inc.), Kanta Matsuura (The Univ. of Tokyo), Atsuko Miyaji (Japan Advanced Inst. of Sci. and Tech.), Koichi Mouri (Ritsumeikan Univ.), Yuko Murayama (Iwate Prefectural Univ.), Toru Nakanishi (Hiroshima Univ.), Masakatsu Nishigaki (Shizuoka Univ.), Ryo Nojima (NICT), Toshihiro Ohigashi (Hiroshima Univ.), Eiji Okamoto (Univ. of Tsukuba), Takeshi Okamoto (Tsukuba Univ. of Tech.), Mirang Park (Kanagawa Inst. of Tech.), Takamichi Saito (Meiji Univ.), Ryoichi Sasaki (Tokyo Denki Univ.), Yuji Suga (IIJ Inc.), Koutarou Suzuki (NTT Corporation), Tsuyoshi Takagi (Kyushu Univ.), Hiroki Takakura (Nagoya Univ.), Kiyoshi Tanaka (Shinshu Univ.), Masato Terada (Hitachi Ltd.), Masayuki Terada (NTT docomo, Inc.), Satoru Tezuka (Tokyo Univ. of Tech.), Satoru Torii (Fujitsu Laboratories Ltd.), Yuji Watanabe (IBM Japan, Ltd.), Toshihiro Yamauchi (Okayama Univ.), Katsunari Yoshioka (Yokohama National Univ.), Hiroshi Yoshiura (Univ. of Electro-Communications)

\footnotetext{
Kanazawa University, Kanazawa, Ishikawa 920-1192, Japan
} 